\title{
Endovascular embolization of ruptured giant pseudoaneurysm of renal angiomyolipoma in a patient with tuberous sclerosis
}

\author{
A Anton Jenil ${ }^{1}$, Balasupramaniam Sathesan², Kukaneson Ajantan², Thangarajah Raviharan², \\ Ketheeswary Sivasethambaram²
}

Ceylon Medical Journal 2019; 64: 118-120

DOI: http://doi.org/10.4038/cmj.v64i2.8953

\section{Introduction}

Tuberous sclerosis (TS) is a neuro-cutaneous disorder characterized by the development of multiple benign tumours. Renal angiomyolipoma (AML) is a benign tumour associated with $55-80 \%$ of patients with TS complex [1]. Typical histology reveals triphasic tumour with myoid spindle cells, mature adipose tissue and dysmorphic thick-walled blood vessels without elastic lamina [3]. Radiologically, it is characterized by the fat content. It is associated with arterial pseudoaneurysm which can lead to life threatening complications. We present a case of ruptured giant pseudoaneurysm with intra capsular hematoma in a renal angiomyolipoma associated with TS.

\section{Case report}

A 34-year-old female with TS and bilateral renal angiomyolipoma presented with acute left flank pain. On admission, she had excruciating pain and was severely pale with haemoglobin of $4.3 \mathrm{~g} / \mathrm{dl}$, requiring urgent blood transfusion. The ultrasound examination was suspicious of left intra-renal bleeding/haematoma. She underwent CT angiogram (Figure 1) following stabilization, which revealed enlargement of both kidneys with multiple angiomyolipomas (largest AML is measuring $6.1 \times 5.4 \mathrm{~cm}$ in the left kidney) associated with left intra-renal pseudoaneurysm, measuring $7.2 \times 5.1 \times 4.2 \mathrm{~cm}$, surrounded by varying ages of haematomas.

A left renal catheter angiogram (Figure 2) confirmed a giant pseudoaneurysm in the lower pole segmental artery with multiple small pseudoaneurysms on the feeder artery.
The patient underwent successful coil (complex helical pushable coils $06 \times 06 \mathrm{~mm}-\mathrm{o} 4)$ and (1000-1180 micron particle) polyvinyl alcohol particle embolization (Figure 3) of the segmental artery. She had an uneventful recovery and was discharged from the hospital a week later.

She had been followed up with monthly ultrasound and her haemoglobin was within normal limit. Her followup CT (Figure 4) at sixth month confirmed the completely occluded left renal pseudoaneurysm. No evidence of recanalization. She is asymptomatic with normal renal profile.

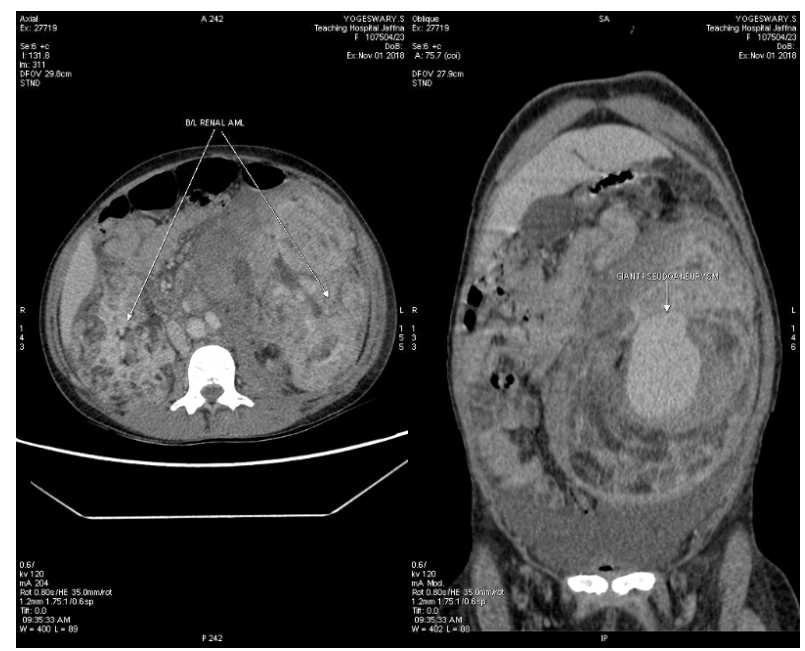

Figure 1. CT angiogram showing bilateral renal multiple angiomyolipomas with left intra-renal pseudoaneurysm. Axial and coronal views.

${ }^{1}$ District General Hospital, Kilinochchi, Sri Lanka, ${ }^{2}$ Teaching Hospital Jaffna, Sri Lanka.

Correspondence: AAJ, e-mail: <aantonjenil@gmail.com>. Received 29 January 2019 and revised version 15 July 2019 accepted 24 September 2019.

(i) This is an open-access article distributed under the terms of the Creative Commons Attribution License, which permits unrestricted use, distribution, and reproduction in any medium, provided the original author and source are credited. 


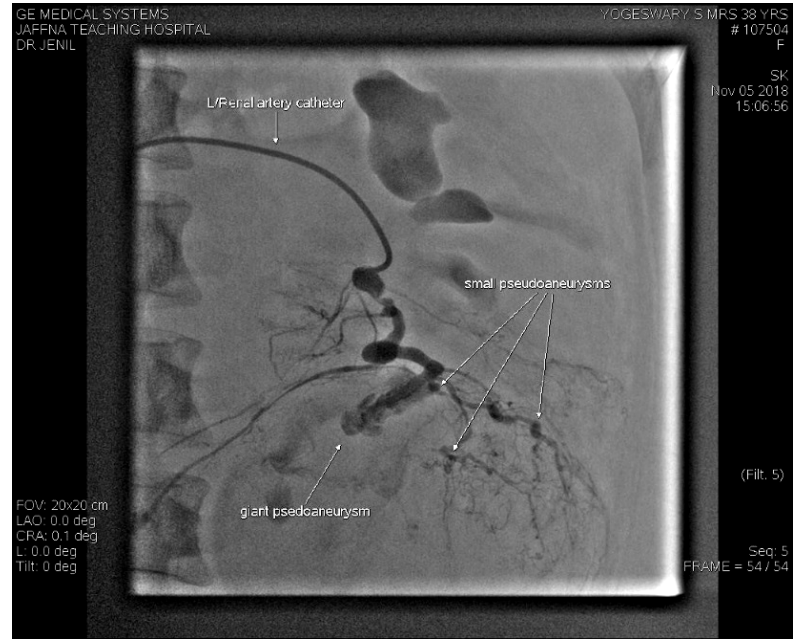

Figure 2. Left renal catheter angiogram demonstrates a giant and multiple small pseudoaneurysms on the feeder artery.

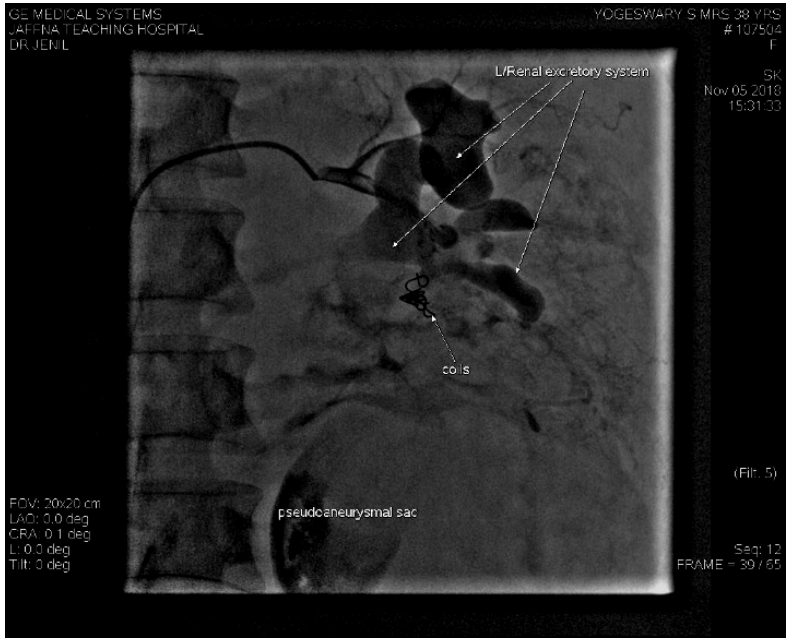

Figure 3. Post embolization angiogram confirmed the successful embolization of the feeder artery.
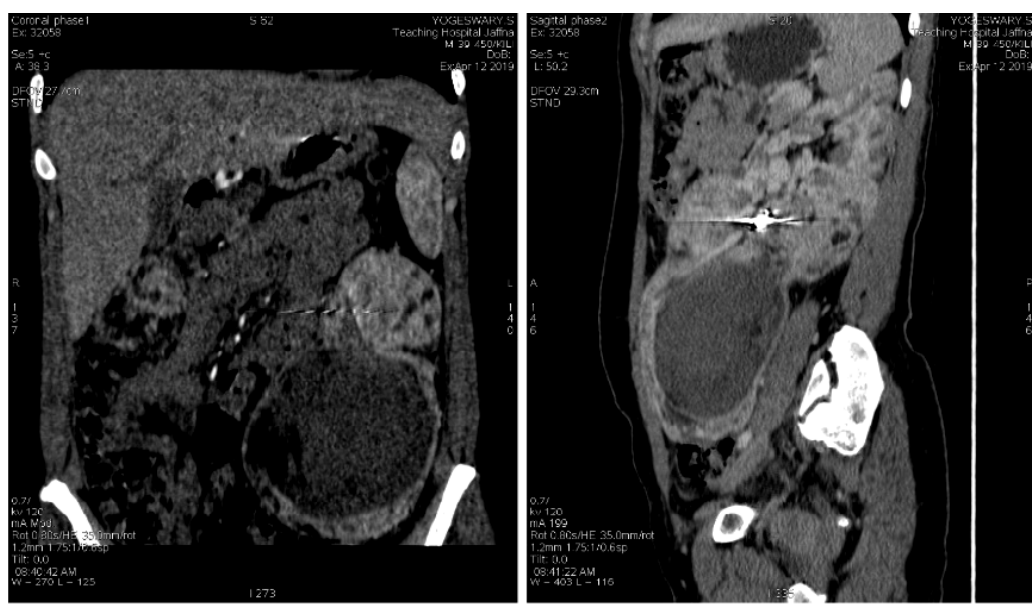

Figure 4. Follow-up CT at sixth month confirmed the completely occluded left renal pseudoaneurysm.

Coronal and sagittal views.

\section{Discussion}

TS related angiomyolipomas are often multiple, bilateral rapidly growing and associated with micro and macro aneurysms predisposing to haemorrhage [2]. The risk of bleeding is higher with tumours larger than $4 \mathrm{~cm}$, rapid tumour growth and aneurysms larger than $0.5 \mathrm{~cm}$ [1]. This patient had a giant aneurysm which ruptured, threatening to her life. Appropriate assessment and immediate intervention prevents fatal outcomes. Selective angiographic embolization is an accepted minimally invasive therapeutic approach which obviates the need for nephrectomy.

\section{Conclusion}

In patients with angiomyolipoma presenting with internal bleeding, a high degree of suspicion of ruptured aneurysm and timely intervention could save lives.

\section{Conflict of interests}

Except I have interested in interventional radiology, no actual or potential conflict of interest exist.

I have no financial disclosures. 


\section{References}

1. Jo-Hoy F, Tolaymat O, Kunjal R, James LR. Tuberous Sclerosis and Bilateral Renal Angiomyolipomas: A Case Report and Literature Review of Emerging Treatment Strategies. Case Rep Nephrol 2016; 2016: 5.

2. Liesbeth De Waele, Lieven Lagae, Djalila Mekahli. Tuberous sclerosis complex: the past and the future. Pediatric Nephrology October 2015; 30: 1771-80.

3. Masahiro Jinzaki, Stuart G. Silverman, Hirotaka Akita, Yoji Nagashima, Shuji Mikami, and Mototsugu Oya. Renal angiomyolipoma: a radiological classification and update on recent developments in diagnosis and management. Abdom Imaging 2014; 39(3): 588-604. 